\title{
CARCINOMA UROTELIAL DA BEXIGA: RELATO DE CASO
}

\author{
Gabriel Bandeira Santos, Felipe lankelevich Baracat, Oscar Rubini Ávila \\ Universidade do Oeste Paulista - UNOESTE, Presidente Prudente, SP. e-mail: gabriel.bandeira santos@hotmail.com
}

\begin{abstract}
RESUMO
Entre os pacientes do sexo masculino, o câncer de bexiga é o quarto mais comum e raramente são identificados em indivíduos jovens. O sintoma manifesto mais comum é a hematúria macroscópica e o tratamento inicial é a ressecção transuretral de bexiga, onde se identifica o estágio e a classificação. Sabe-se que o tabagismo é o principal fator de risco para os cânceres de bexiga. A cistectomia radical e a linfadenectomia bilateral pélvica são o tratamento "padrão ouro" para o câncer de bexiga musculo-invasivo. Cerca de $25 \%$ das neoplasias de bexiga são músculo-invasivas no momento do diagnóstico, mas os pacientes têm um bom prognóstico com esse padrão de tratamento. A técnica de Bricker é a mais usada para a reconstrução da neobexiga. Nesse artigo relatamos o caso de um paciente de 55 anos, com câncer de bexiga invasivo de alto grau, sendo tratado com uma cistectomia radical, linfadenectomia e neobexiga usando a técnica de Bricker. Após o procedimento cirúrgico, o paciente permaneceu dois dias na Unidade de Tratamento Intensivo, evoluindo com insuficiência renal aguda e necessidade de três sessões de hemodiálise. A recuperação foi adequada, apresentando uma boa evolução pós-operatória. Enfatizamos a importância de relatar para o paciente sobre o indispensável acompanhamento clínico, que deve ser realizado até que seja dada a alta médica, para evitar a recidiva da doença e necessidade de tratamentos mais agressivos, como veremos neste caso.
\end{abstract}

Palavras-chave: urologia, neoplasias da bexiga urinária, urotélio, hematúria, cistectomia.

\section{UROTHELIAL BLADDER CARCINOMA: CASE REPORT}

\begin{abstract}
Among male patients, bladder cancer is the fourth most common and is rarely identified in young individuals. The most common symptom is macroscopic hematuria and the initial treatment is transurethral resection of the bladder, in which the stage and classification are identified. Smoking is known to be the main risk factor for bladder cancers. Radical cystectomy and bilateral pelvic lymphadenectomy are the "gold standard" treatment for muscle-invasive bladder cancer. Around $25 \%$ of bladder neoplasms are muscle-invasive at diagnosis, but the patients have a good prognosis with this kind of treatment. The Bricker technique is the most used for the reconstruction of the neobladder. In this paper we report the case of a male patient, 55 years old, with invasive high-grade bladder cancer, treated with a radical cystectomy, lymphadenectomy and neobladder using the Bricker technique. After the surgical procedure, the patient remained in the Intensive Care Unit for two days, presenting acute kidney injury and the need for three hemodialysis sessions. The recovery was adequate, with a good postoperative evolution. We emphasize the importance of reporting to the patient about the indispensable clinical follow-up, which must be done until medical discharge, to avoid disease recurrence and the need for more aggressive treatments, as we will see in this case.
\end{abstract}

Keywords: urology, urinary bladder neoplasms, urothelium, hematuria, cystectomy. 


\section{INTRODUÇÃO}

O câncer de bexiga é o quarto câncer mais comum em homens e o nono em mulheres, nos Estados Unidos ${ }^{1}$. São estimados cerca de 81.190 novos casos para 2018, com 17.240 mortes, sendo elas, 12.520 em homens $^{2}$. Segundo o INCA (Instituto Nacional de Câncer), são previstos 9.480 novos casos de carcinoma urotelial para 2018 , sendo $70,5 \%$ em homens e $29,5 \%$ em mulheres. O Sistema de Informação de Mortalidade refere que houve 3.634 mortes em 2013 relacionadas a esta doença, no Brasil. Este tumor possui uma baixa frequência em pessoas menores de quarenta anos de idade, sendo a média de diagnóstico de 65 anos $^{1}$. Os carcinomas uroteliais podem se desenvolver em qualquer lugar onde o epitélio de transição esteja presente, entre a pelve renal e os dois terços proximais da uretra, sendo a bexiga responsável por mais de $90 \%$ deles $^{1}$.

O sintoma mais comum em pacientes com câncer de bexiga é a hematúria microscópica ou macroscópica, embora a diminuição da frequência urinária ou a capacidade reduzida da bexiga também possam se desenvolver. As infecções do trato urinário são muito raras, ao passo que a obstrução do trato urinário ou a dor podem ocorrer em uma lesão mais avançada. Os pacientes que apresentam esses sintomas devem ser avaliados por meio de cistoscopia. Caso haja lesão detectada, o paciente deve ser encaminhado para uma ressecção transuretral (RTU) do tumor da bexiga, para confirmar o diagnóstico e determinar a extensão da doença dentro da bexiga ${ }^{1}$.

A RTU de bexiga é o tratamento inicial para todas as suspeitas de câncer de bexiga. $\mathrm{O}$ objetivo da RTU é identificar corretamente o estágio e, para tanto, o músculo da bexiga deve ser incluído nas biópsias da ressecção ${ }^{1}$. Avanços recentes na ressecção transuretral do tumor da bexiga auxiliam na precisão diagnóstica, ajudam a reduzir recorrências, a diminuir complicações e a reduzir o custo do tratamento do câncer de bexiga não musculo-invasivo ${ }^{3}$.

A cistectomia radical (CR) e a linfadenectomia bilateral pélvica continuam a ser o tratamento "padrão ouro" para câncer de bexiga musculo-invasivo com alto risco e os superficiais resistentes à terapia intravesical ${ }^{4,5}$. Vários estudos demonstraram excelentes taxas de sobrevida em cinco e dez anos após a $\mathrm{CR}^{5}$. A sobrevida global aos 3 anos foi de $54 \%$ e aos 5 anos foi de $48 \%$ nos pacientes com manifestações extravesicais e, os casos com doença localizada à bexiga apresentaram uma sobrevida aos 3 anos de $89 \%$, segundo um renomado estudo retrospectivo ${ }^{6}$. A cistectomia radical fornece informações de estadiamento precisas, que são a chave para a decisão em relação às opções de tratamento adjuvante, enquanto o estadiamento por meios clínicos está associado a um erro de estadiamento de até $30 \%{ }^{7}$.

Simultaneamente ao tratamento cirúrgico radical, surge a necessidade de reconstrução de uma neobexiga. A técnica mais utilizada no Brasil é a derivação de Bricker. Nesta cirurgia, é selecionado um segmento do íleo com 10 a 15 centímetros de comprimento à cerca de quinze centímetros da válvula íleocecal. Esse segmento será a neobexiga, onde será feita a anastomose dos ureteres ao conduto ileal. $\mathrm{O}$ estoma ficará no quadrante inferior direito do abdome, havendo a necessidade de utilização de uma bolsa coletora ${ }^{8}$.

Neste artigo, relatamos o caso de um paciente do sexo masculino com câncer de bexiga invasivo de alto grau e a sua evolução, assim como buscamos trazer o que há de mais atual no manejo adequado deste tumor, já que a doença revelou uma importante agressividade, pela invasão muscular e angiolinfática.

\section{RELATO DE CASO}

Paciente do sexo masculino, 55 anos, hipertenso, tabagista e etilista, admitido em um hospital público terciário com hematúria macroscópica há 4 dias, fraqueza e dispneia há 2 dias. O paciente referiu história prévia de tumor de bexiga há 4 anos, sendo tratado cirurgicamente em outro serviço com perda de seguimento. A tomografia computadorizada demonstrou irregularidades de contorno no assoalho vesical.

Foi, então, internado para a realização da RTU de bexiga, que evidenciou a lesão próxima ao meato ureteral esquerdo, teto vesical e parede lateral direita, sendo ressecados vários fragmentos medindo em conjunto $8,0 \times 6,3 \times 4,0 \mathrm{~cm}$, de coloração castanha clara e consistência firme. A RTU foi interrompida devido ao tamanho das lesões e intenso sangramento, optando-se por proceder com hemostasia e reprogramar nova abordagem para retirada do restante da lesão.

A microscopia revelou neoplasia de crescimento infiltrativo, constituída por células epiteliais anaplásicas exibindo acentuado grau de hipercromasia e pleomorfismo nuclear. O diagnóstico foi de carcinoma urotelial papilífero de alto grau, infiltrando o córion e não observando invasão de túnica muscular. 
Um mês após, o paciente retornou para nova RTU de bexiga. O novo material ressecado revelou uma importante evolução no diagnóstico anatomopatológico: carcinoma urotelial papilífero de alto grau, com infiltração nas camadas muscular da mucosa e muscular própria, além de infiltração angiolinfática.

Decidiu-se, então, pela realização de uma cistoprostatectomia radical com neobexiga à Bricker e linfadenectomia ilíaca.

O paciente permaneceu dois dias na UTI (Unidade de Terapia Intensiva), evoluindo com insuficiência renal aguda, com necessidade da realização de hemodiálise em três episódios, recuperando a função renal e apresentando diurese satisfatória. Logo após a alta hospitalar, aos dezenove dias de pós-operatório, ocorreu deiscência da ferida operatória, passível de tratamento clínico, tendo o paciente recebido alta hospitalar após 9 dias.

Este relato de caso foi aprovado pelo Comitê de Ética em Pesquisa da Universidade do Oeste Paulista (CAAE no 04386818.7.0000.5515).

\section{DISCUSSÃO}

Carcinomas uroteliais têm múltiplas variantes morfológicas com diferentes implicações terapêuticas e prognósticas ${ }^{9}$. De acordo com a American Cancer Society, $90 \%$ dos cânceres de bexiga ocorrem em indivíduos acima dos 55 anos e o tabagismo é o fator de risco mais importante, responsável por metade dos casos. O carcinoma urotelial invasivo de bexiga é uma doença potencialmente letal que requer tratamento agressivo e, menos de $15 \%$ dos pacientes sobrevivem dois anos se não tratados. A cistectomia radical permanece como principal tratamento para o tumor de bexiga com invasão da camada muscular. Confirmada a invasão muscular após a RTU, a cistectomia deve ser realizada o mais breve possível. Há evidência de que uma demora maior do que três meses entre a ressecção transuretral e a cistectomia altera o estadiamento, piorando o prognóstico ${ }^{10}$. 0 paciente relatado não obteve sucesso no tratamento logo após o diagnóstico, resultando na recidiva do tumor, após a perda do seguimento. Ao procurar o serviço médico novamente, houve a necessidade da realização de duas RTU de bexiga, tendo um intervalo de um mês entre elas. Foi, então, realizado o tratamento cirúrgico logo em seguida, para evitar a piora prognóstica.

O procedimento padrão consiste em linfadenectomia pélvica bilateral associada à cistectomia radical, no homem. Nos pacientes com câncer invasor de muscular, 25\% apresentam metástases em linfonodos e pior prognóstico. O objetivo é realizar uma ampla ressecção, incluindo toda a gordura perivesical e tecidos adjacentes, para se obter margens cirúrgicas negativas. A linfadenectomia recomendada consiste na remoção de todos os linfonodos situados na porção distal das artérias ilíacas comuns, hipogástricas, obturadoras e ilíacas externas, o que resulta em 10 a 14 linfonodos ${ }^{10}$. Já existem evidências relevantes de que a extensão da linfadenectomia é um importante fator de cura no câncer de bexiga ${ }^{11}$.

A segunda RTU de bexiga foi mais eficaz, em relação a primeira, quanto a coleta do material ressecado, o que resultou em uma importante evolução no resultado do anatomopatológico, agora com invasão nas camadas muscular da mucosa e muscular própria, além de infiltração angiolinfática. Após a cistoprostatectomia do paciente, os achados foram: focos de carcinoma in situ na mucosa adjacente; parênquima prostático, uretra, as margens cirúrgicas dos ureteres, vesículas seminais, linfonodos inguinais e demais margens cirúrgicas livres de neoplasias, concluindo o estadiamento anatomopatológico como pT2A pNO, segundo a Classificação TNM (Tumor Nódulo Metástases) 2009 para câncer de bexiga ${ }^{12}$.

Vários avanços recentes contribuíram para diminuir a morbidade, melhorar a sobrevida e a qualidade de vida após o cistectomia radical, que incluem: cistectomia radical laparoscópica e assistida por robô, modelos estendidos de linfadenectomia, aumento do uso de derivação urinária continente, cistectomia radical poupadora de nervos, quimioterapia neoadjuvante e adjuvante ${ }^{5}$. Dentre as complicações pós-operatórias da CR, as deiscências das suturas constituem $15 \%$ dentre todas ${ }^{13}$.

Existem diversas técnicas de derivações urinárias, que devem aproximar-se ao máximo da bexiga normal. A derivação de Bricker é a mais usada em todo o mundo, pela sua menor complexidade cirúrgica, menores índices de complicações pósoperatórias e melhor preservação da função renal de longo prazo, pois funcionam como um conduto. A desvantagem é o uso de bolsa urinária de urostomia definitivamente ${ }^{8}$.

Este caso é valoroso, para a prática médica, no sentido de perceber a relevância de enfatizar para o paciente sobre a importância de manter o correto acompanhamento clínico da doença até que seja dada a alta médica. Isto evita, por exemplo, a necessidade de um tratamento mais agressivo ao diagnosticar tardiamente uma recidiva, como aconteceu com o paciente relatado, evita maiores 
danos à saúde do paciente e maiores gastos ao sistema público de saúde. Este artigo é pertinente, também, para o conhecimento médico sobre o carcinoma urotelial de bexiga, pois reuniu pontos importantes do adequado manejo clínico do paciente, desde a formulação diagnóstica até a discussão da melhor abordagem cirúrgica para o tratamento desta enfermidade.

Em geral, pacientes pós-cistectomia radical tendem a ter um desfecho favorável. Um quarto dos pacientes com câncer de bexiga é invasivo no momento do diagnóstico e um terço dos tumores não invasivos progridem para invasivo, mostrando a importância deste relato, para definir o manejo correto desses casos. O tratamento padrão para o câncer de bexiga músculo invasivo da muscular é a cistectomia radical com linfadenectomia pélvica bilateral. A linfadenectomia tem um papel importante no diagnóstico, no prognóstico dos pacientes e definição do tratamento adjuvante.

\section{CONFLITO DE INTERESSE}

Os autores declaram não haver qualquer potencial conflito de interesse que possa interferir na imparcialidade deste trabalho científico.

\section{REFERÊNCIAS}

1. Clark PE, Agarwal N, Biagioli MC, Eisenberger MA, Greenberg RE, Herr HW et al. Bladder cancer. J Natl Compr Canc Netw. 2013;11(4):446-76. DOI: https://doi.org/10.6004/inccn.2013.0059

2. Siegel RL, Miller KD, Jemal A. Cancer statistics, 2018. CA Cancer J Clin. 2018;68(1):7-30. DOI: https://doi.org/10.3322/caac.21442

3. Richards K.A, Smith N.D, Steinberg G.D. The importance of transurethral resection of bladder tumor in the management of nonmuscle invasive bladder cancer: a systematic review of novel technologies. J Urol. 2014;191(6):1655-64. DOI: https://doi.org/10.1016/j.juro.2014.01.087

4. Simonato A, Gregori A, Lissiani A, Bozzola A, Galli S, Gaboardi F. A Technique Illustrated Step by Step. Eur Urol. 2003;44(1):132-8. DOI: https://doi.org/10.1016/S0302-2838(03)00214-8

5. Gupta NP, Kolla SB, Seth A, Droga PN, Hemal AK, Kumar $R$ et al. Radical cystectomy for bladder cancer: A single center experience. Indian J Urol. 2008;24(1):54-9.

DOI:
6. Araújo P, Cruz R, Freitas R, Morais A, Oliveira J. Radical cystectomy / experience of an oncology center. Instituto Português de Oncologia do Porto, Portugal, 2014.2 DOI: http://dx.doi.org/10.1016/S2341-4022(14)50052-3

7. Ghoneim MA, El-Mekresh MM, El-Braz MA, ElAttar IA, Ashamallah A. Radical cystectomy for carcinoma of the bladder: critical evaluation of the results in 1,026 cases. J Urol. 1997;158(2):393-9. DOI: https://doi.org/10.1016/S00225347(01)64487-2

8. Nardi AC, Júnior AN, Bezerra CA, Fonseca CEC, Truzzi JC, Rios LAS et al. Urologia Brasil. São Paulo: Planmark; 2013

9. Oliveira RP, Abrantes CF, Silva EM, Marinho CA, Sousa VM, Castro LR. Carcinoma urotelial invasor, variante plasmocitoide: relato de caso. J Bras Patol. Med Lab. 2016;52(4):262-265. DOI: http://dx.doi.org/10.5935/1676-2444.20160043

10. Stein JP, Lieskovsky G, Cote R, Groshen S, Feng A, Boyd $S$ et al. Radical cystectomy in the treatment of invasive bladder cancer: long-term results in 1,054 patients. J Clin Oncol. 2001;19(3):666-75. DOI: https://doi.org/10.1200/JCO.2001.19.3.666

11. Danbgni G, Geneca E, Hashibe M, Zhang Z, Russo $\mathrm{P}$, Herr $\mathrm{H}$ et al. Cystectomy for bladder cancer: a contemporary series. J Urol. 2001;165(4):1111-6. DOI: $\quad$ https://doi.org/10.1016/S00225347(05)66440-3

12. Chisholm GD, Hindmarsh JR, Howatson AG, Webb JN, Bussutil A, Hargreave TB et al. TNM (1978) in Bladder Cancer. $\mathrm{Br} J$ Urol. 1980;52(6):500-5. $1980 . \quad$ DOI: https://doi.org/10.1111/j.1464-410X.1980.tb03100.x

13. Liedberg F. Early complications and morbidity of radical cystectomy. Eur Urol Suppl. 2010;9(1):25-30. DOI: https://doi.org/10.1016/j.eursup.2010.01.007

http://dx.doi.org/10.4103/0970-1591.38604 